\title{
On the dependence of planetary spin on mass
}

\author{
Francesco Meneguzzo*, Lorenzo Albanese \\ Institute of Biometeorology, National Research Council, Firenze, 8 Via Caproni, Italy \\ Email address: \\ f.meneguzzo@ibimet.cnr.it (F. Meneguzzo), 1.albanese@ibimet.cnr.it (L. Albanese)
}

\section{To cite this article:}

Francesco Meneguzzo, Lorenzo Albanese. On the Dependence of Planetary Spin on Mass. American Journal of Astronomy and Astrophysics. Vol. 2, No. 3, 2014, pp. 27-33. doi: 10.11648/j.ajaa.20140203.11

\begin{abstract}
The recent direct spectroscopic observation of the spin of the young gas giant exoplanet $\beta$ Pictoris $b$ was a powerful clue as to the general validity of the trend of the planetary spin with the mass even outside the Solar System. Nevertheless, the spin-mass relationship, which looks like to hold irrespective of the planet composition and radius, is admittedly poorly understood. On the basis of bilogarithmic regressions, the rotational kinetic energy is found to explain the available data more significantly than the equatorial rotation velocity but no more than the spin angular momentum; nevertheless, only the rotational energy turns out to be closely proportional to the square of the mass of planets, suggesting its possible close and direct ties to the planetary mass by means of some fundamental processes. The hypothesis is made that such underlying physical processes can be described by the non-gauge cosmological theory of byuons, which proved useful to explain other astrophysical and geophysical puzzling phenomena such as the motion of pulsars, the nature of dark matter and dark energy, the anisotropy of cosmic rays and the accelerated expansion of the Universe. It's shown that the theory of byuons is able to explain the observed close proportionality of the planetary rotational kinetic energy to the square of the mass.
\end{abstract}

Keywords: $\beta$ Pictoris b, Planetary Spin, Spin Angular Momentum, Rotational Kinetic Energy, Planetary Mass, Theory of Byuons

\section{Introduction}

The existence of some relation between planetary spin and mass was noticed decades ago; the most accurate and significant empirical result for the four gas giant planets in the Solar System is likely the approximate proportionality of the their spin angular momenta with the squares of the respective masses, such relation holding as well for the other planets except for Mercury and Venus, which spin has probably been decreased by the tidal interactions with the nearby Sun [1].

The quantitative assessment of the spin of the young extrasolar gas giant planet $\beta$ Pictoris $b$ based for the first time on direct spectroscopic observations was recently reported [2]. Representing the planetary spin by means of the planet's equatorial rotation velocity, $\beta$ Pictoris $b$ is shown to spin significantly faster than any planet in the Solar System, in agreement with the observed trend in spin velocity with planet mass. Although their analysis is confined to the equatorial rotation velocity (see Fig. 2 in [2]), therefore missing to check the long known relationship between planetary spin angular momentum and square of the mass, while the general trend of the spin with the mass is unlikely to have occurred by chance, it would reflect important, but admittedly as yet poorly understood, aspects of how the planets formed, as pointed out as well in a review of the original paper [3]. Clues of the uncertainty surrounding the mechanisms leading to the increase of the planetary spin with the mass arises from statements found in [2] and in [3]: in the first one, it is stated that the origin of the spin-mass relation must be linked to the mass accretion processes that occurred during planet formation, while according to the second paper impacts with large bodies, which impart significant angular momentum to the planet, may have a crucial role in the late stages of planet formation across the full range of planet masses.

In any case and beyond the details, the conservation of the angular momentum from substance building up the planet or hitting the planet at some time of its formation and growth looks like to be the generally accepted process finally leading to the observed planetary spin.

While the conservation of the angular momentum is of course an universal rule of physics at least in the macroscopic world and the above mentioned processes cannot be ruled out, significant clues about the very origin of the energy available for planetary rotation are suggested 
on the basis of a non-gauge cosmological theory, which is proved to provide a direct and accurate explanation of the observed data.

The paper is organized as follows: Section 2 shortly describes data and methods; Section 3 introduces the theoretical background meant to explain the origin of the planetary rotational energy; in Section 4 the results from observational data are first derived, then compared with the theoretical predictions and discussed. Conclusions and perspectives for further work are available in Section 5.

\section{Data and Methods}

The spin angular momentum $(\Pi)$ and the planetary rotational kinetic energy $(R K E)$ are represented by the usual expressions:

$$
\begin{aligned}
\Pi & =I_{z} \omega \\
R K E & =(1 / 2) I_{z} \omega^{2}
\end{aligned}
$$

where $I_{z}$ is the planetary moment of inertia, $\omega=v / r$ is the angular velocity, $v$ is the equatorial rotation velocity and $r$ is the radius.

Mass, radius, moment of inertia and equatorial rotation velocity for Solar System planets were drawn from [4]; missing more exact data, the upper bound value for Neptune's $I_{z}$ [4] and the average value across a range for Pluto's $I_{z}[5]$ were used.

Mass, radius and equatorial rotation velocity for $\beta$ Pictoris b were drawn from [2], while its moment of inertia was assumed to scale with the mass and the square radius as for Jupiter due to their close similarity [2,6]; uncertainties about its mass and radius were as well drawn from [2].

The whole list of data used in this report is available in Table 1, where the moment of inertia is given in terms of its multiplicative factor for mass and square radius, i.e. $k=$ $I_{z} / m r^{2}$, where $m$ is the planet's mass.

The quantitative relationships of the equatorial rotation velocity ( $E R V), R K E$ and $\Pi$ with the mass of the considered planets are assessed on the basis of bilogarithmic regressions, as was done in [1] and [2].

\section{At the Origin of Planetary Rotation}

The cosmological theory of byuons, a non-gauge theory of the formation of the physical space and the elementary particles from unobservable objects called byuons [7-11], was proved to offer useful interpretation or shed some new light on puzzling astrophysical phenomena such as the motion of pulsars, dark matter and dark energy, the accelerating expansion of the Universe and the anisotropy of cosmic rays [12-14], as well as on few geophysical processes, such as earthquakes [15] and the atmospheric tornadoes characterized by unusual rotational energy [16]. In $[8,9]$ the earliest experiments are described where a fundamental anisotropy of the physical space as well as a new non-gauge interaction, different from the four known ones (strong, weak, electromagnetic, and gravitational), were first detected. Besides the lack of gauge invariance, an important feature of this new interaction is just its anisotropy that arises in a wide range of sizes, from the weak interactions, i.e. $10^{-19} \mathrm{~m}$ [17], up to the size of our Galaxy, i.e. $10^{21} \mathrm{~m}$ [12], and more [14].

The physical nature of the new force is explained by the byuon theory $[13,18,19]$, according to which the potentials of physical fields can act upon the process of mass formation of the elementary particles [11], because a fraction of such mass, associated with the formation of their inner space, is proportional to the modulus of a summary potential $A_{\Sigma}$ i.e. the sum of the potentials of all known force fields calculated using the energy relation expressed by equation (3.1.) in Baurov and Malov [20]. Such summary potential cannot exceed, by magnitude, the modulus of the cosmological vectorial potential $\mathrm{A}_{\mathrm{g}}$, a new fundamental constant having absolute value $\left|\mathrm{A}_{\mathrm{g}}\right| \approx 1.9 \cdot 10^{5} \mathrm{~T} \cdot \mathrm{m}$.

Table 1. Planetary data. References for data sources, as well as the expression for $\Pi$ (equation (1)) and RKE (equation (2)), are available in the main text. $\beta$ Pictoris b: uncertainties are given only for mass and radius, while no uncertainty figure is available for the equatorial rotation velocity and computed

\begin{tabular}{|c|c|c|c|c|c|c|c|}
\hline Planet & $\begin{array}{l}\text { Mass } \\
(\mathrm{kg})\end{array}$ & $\begin{array}{l}\text { Radius } \\
\text { (m) }\end{array}$ & $\begin{array}{l}\text { Equatorial rotation } \\
\text { velocity }\left(\mathrm{m} \mathrm{s}^{-1}\right)\end{array}$ & $\begin{array}{l}\text { Angular velocity } \\
\left(\mathrm{rad} \mathrm{s}^{-1}\right)\end{array}$ & $\begin{array}{l}\text { Moment of inertia } \\
\left(\mathbf{k}=\mathbf{I}_{\mathbf{z}} / \mathbf{m} \mathbf{R}^{\mathbf{2}}\right)\end{array}$ & $\begin{array}{l}\text { RKE } \\
\text { (J) }\end{array}$ & $\begin{array}{l}\Pi \\
\left(\mathrm{kg} \mathrm{m}^{2} \mathrm{~s}^{-1}\right)\end{array}$ \\
\hline Earth & $5.97 \cdot 10^{24}$ & $6.37 \cdot 10^{6}$ & 465 & $7.29 \cdot 10^{-05}$ & 0.3308 & $2.13 \cdot 10^{29}$ & $5.85 \cdot 10^{33}$ \\
\hline Mars & $6.42 \cdot 10^{23}$ & $3.39 \cdot 10^{6}$ & 240 & $7.09 \cdot 10^{-05}$ & 0.3660 & $6.78 \cdot 10^{27}$ & $1.91 \cdot 10^{32}$ \\
\hline Jupiter & $1.90 \cdot 10^{27}$ & $7.12 \cdot 10^{7}$ & 13,378 & $1.88 \cdot 10^{-04}$ & 0.2540 & $4.31 \cdot 10^{34}$ & $4.59 \cdot 10^{38}$ \\
\hline Saturn & $5.68 \cdot 10^{26}$ & $6.00 \cdot 10^{7}$ & 9,830 & $1.64 \cdot 10^{-04}$ & 0.2100 & $5.77 \cdot 10^{33}$ & $7.04 \cdot 10^{37}$ \\
\hline Uranus & $8.68 \cdot 10^{25}$ & $2.45 \cdot 10^{7}$ & 2,477 & $1.01 \cdot 10^{-04}$ & 0.2250 & $5.99 \cdot 10^{31}$ & $1.18 \cdot 10^{36}$ \\
\hline Neptune & $1.03 \cdot 10^{26}$ & $2.50 \cdot 10^{7}$ & 2,712 & $1.08 \cdot 10^{-04}$ & 0.2390 & $9.05 \cdot 10^{31}$ & $1.67 \cdot 10^{36}$ \\
\hline Pluto & $1.32 \cdot 10^{22}$ & $1.16 \cdot 10^{6}$ & 13 & $1.14 \cdot 10^{-05}$ & 0.3500 & $4.03 \cdot 10^{23}$ & $7.07 \cdot 10^{28}$ \\
\hline$\beta$ Pictoris $b$ & $\begin{array}{l}2.09 \cdot 10^{28} \\
\pm 9.49 \cdot 10^{27}\end{array}$ & $\begin{array}{l}1.18 \cdot 10^{8} \\
\pm 4.27 \cdot 10^{6}\end{array}$ & 25,324 & $2.15 \cdot 10^{-04}$ & 0.2540 & $1.70 \cdot 10^{36}$ & $1.58 \cdot 10^{40}$ \\
\hline
\end{tabular}
errors for RKE and Пare shown graphically in the charts. 
As the result of the action of the field potentials (decreasing $\left|\mathrm{A}_{\Sigma}\right|$ ), each particle gains an energy $\Delta \mathrm{m} \cdot \mathrm{c}^{2}$ that corresponds to a new force of nature throwing substance out of the region with the weakened $A_{\Sigma}$. Experimental investigations with the use of gravimeters and magnets and plasma systems [7], as well as the measurements of changes in the $\beta$-decay rate of radioactive elements $[17,21]$, that were later confirmed by independent researches [22], have shown that the substance is ejected from the region with the weakened $A_{\Sigma}$ along a cone with an angular opening about $100^{\circ}$ around the vector $A_{g}$ determining the global anisotropy of the physical space and having the following astronomical coordinates in the second equatorial system: $\alpha \approx 300^{\circ} \pm 10^{\circ}, \delta \approx 36^{\circ} \pm 10^{\circ}$, where $\alpha$ is the right ascension and $\delta$ is the declination [23].

The analysis of a long run of experiments has shown that the new force has a nonlinear and nonlocal character and can be represented as a complex series in terms of changes of the summary potential $A_{\Sigma}$. The first term of the series is the following:

$$
\mathrm{F}=2 \mathrm{Nm}_{\mathrm{v}} \mathrm{c}^{2} \lambda_{1}^{2} \Delta \mathrm{A}_{\Sigma}\left[\Delta\left(\Delta \mathrm{A}_{\Sigma}\right) / \Delta \mathrm{x}\right]
$$

where $\mathrm{N}$ is the number of stable particles (electrons, protons, and neutrons) in the test body, $\Delta \mathrm{A}_{\Sigma}$ is the difference in changes of the summary potential $A_{\Sigma}$ at the location points of a test body and sensor element, $\Delta\left(\Delta \mathrm{A}_{\Sigma}\right) / \Delta \mathrm{x}$ is the gradient in space of the difference potentials $\Delta \mathrm{A}_{\Sigma} ; \mathrm{x}$ is the length of an arc of a circle for experiments with solenoids, therefore a space coordinate; $2 \mathrm{Nm}_{\mathrm{v}} \mathrm{c}^{2}=33 \mathrm{eV} ; \lambda_{1}=10^{-6}(\mathrm{Tm})^{-1}$ is the first coefficient of the series.

The rotational energy for any natural macroscopic objects (i.e. objects in an $\mathrm{R}_{3}$ space) is predicted by the theory of byuons to arise as a fundamental result of the minimization of the potential energy of the byuon interactions in special vacuum states (see page 26 in [13]).

In order to estimate the onset of the kinetic rotational energy of a planet during its formation, the following variable factors of the first order term of the force in (3) are considered:

$$
\mathrm{F} \sim \mathrm{N} \cdot \Delta \mathrm{A}_{\Sigma} \cdot\left[\Delta\left(\Delta \mathrm{A}_{\Sigma}\right) / \Delta \mathrm{x}\right]
$$

In (4), $\mathrm{N}$ is proportional to the mass $\mathrm{m}$ of the planet at any stage of its formation, when the planet's radius is $r$, .i.e. $\mathrm{N} \sim \mathrm{m}(\mathrm{r})$.

The large scale change of the summary potential $\Delta \mathrm{A}_{\Sigma}$ will be due to the changes of the gravitational potential produced by some remote large source such as the Sun or the specific star (as is the case for $\beta$ Pictoris $b$ ) and due to the changing distance between a planet and its star during the planet's revolution, such as represented by equation (3.2) and equation (3.3) in [20], which is independent of the planet's mass.

The spatial gradient of the summary potential, i.e. the factor $\Delta\left(\Delta \mathrm{A}_{\Sigma}\right) / \Delta \mathrm{x}$, arises as a consequence the changes of the planet's own gravitational potential in turn proportional to the irregular accumulation of its mass during the planet's formation, namely $\delta \mathrm{m}(\mathrm{r})$, hence: $\Delta\left(\Delta \mathrm{A}_{\Sigma}\right) / \Delta \mathrm{x} \sim \delta \mathrm{m}(\mathrm{r}) / \Delta \mathrm{x}$.

The work $\delta \mathrm{W}(\mathrm{r})$ performed by the force represented in (4) at some stage of planet's formation will be given by such force times the characteristic length scale of its action, the latter quantity corresponding to the same length scale $\Delta \mathrm{x}$ of the spatial gradient of the perturbation to the summary potential, due to the nature of the new force (the force exists in the same region where such spatial gradient is non zero):

$$
\delta \mathrm{W}(\mathrm{r}) \sim \mathrm{F} \cdot \Delta \mathrm{x} \sim \mathrm{m}(\mathrm{r}) \cdot[\delta \mathrm{m} / \Delta \mathrm{x}] \cdot \Delta \mathrm{x} \sim \mathrm{m}(\mathrm{r}) \cdot \delta \mathrm{m}
$$

Since the planet's radius $r$ has no role in any work performed by the force $\mathrm{F}$ at any stage of the planet's formation, the partial works given by (5) can be straightforwardly integrated over the planet's mass:

$$
\mathrm{W} \sim \int_{0}^{M} m d m \sim \mathrm{M}^{2}
$$

where $\mathrm{M}$ is the final planet's mass, while $\mathrm{W}$ is the total work generating the rotation that, by the usual energy conservation law, must equal the rotational kinetic energy shown by the planet after its complete formation.

Overall, once it's assumed that most of the residual potential energy of byuon interaction turns into rotational energy, further accounting for the physical forcing of the new force and finally performing a simple scale analysis in the physical spatial domain, the basic result is obtained, that the rotational kinetic energy of a planet should be proportional to the square of its own mass, regardless of its radius, substance and density profile.

For objects like stars or pulsars, having as well huge magnetic potentials [12], the assessment of the spatial gradient of the summary potential $\Delta\left(\Delta \mathrm{A}_{\Sigma}\right) / \Delta \mathrm{x}$ becomes much more complicated and outside the scope of this article.

\section{Results and Discussion}

The predictive ability of the bilogarithmic regressions of ERV, $\Pi$ and RKE as functions of the masses of Solar System planets listed in Table 1 with regards to the planet $\beta$ Pictoris b is assessed in Fig. 1a, Fig. 1b and Fig. 1c, respectively.

While the $E R V$ regression produces a very significant overestimation for $\beta$ Pictoris $b$, as already noticed elsewhere [2], both its $\Pi$ and $R K E$ appear to be very well predicted by means of the respective regressions performed using the data of planets in the Solar System, especially when its mass uncertainty is taken into account. The marginal superiority shown by the spin angular momentum (Fig. 1b) over the rotational kinetic energy (Fig. 1c) disappears when data for Pluto are excluded (not shown), which may be justified by its small mass (about one sixth that of Earth's Moon) and by the binary system it belongs 
to with Charon, a nearby celestial body having a radius just over half that of Pluto itself.

The close similarity of the predictive performances of $\Pi$ and $R K E$ is quite obvious because such quantities are built on the basis of the same data, i.e. the moment of inertia $I_{z}$ and the angular velocity $\omega$, as well as their logarithms are linear combinations of the logarithms of $I_{z}$ and $\omega$.

On the basis of the demonstrated predictive ability of the regressions of the planetary $\Pi$ and $R K E$ on the masses of the Solar system planets, data from $\beta$ Pictoris $b$ were added in order to improve the statistics. The results are shown in Fig. 2(a) and Fig. 2(b), respectively.

The bilogarithmic regression equations including data from $\beta$ Pictoris $b$ are as follows:

$$
\begin{gathered}
\log _{10}(\Pi)=(1.85 \pm 0.06) \log _{10}(M)-(12.1 \pm 1.4), \\
\mathrm{R}^{2}=99.7 \%
\end{gathered}
$$

$$
\begin{gathered}
\log _{10}(R K E)=(2.05 \pm 0.08) \log _{10}(M)-(21.3 \pm 2.0), \\
\mathrm{R}^{2}=99.5 \%
\end{gathered}
$$

where $\mathrm{M}$ is the planet's mass and $\mathrm{R}^{2}$ is the percentage of the explained variance.

The errors associated with the regression coefficients were computed accounting for the uncertainties of the mass and radius of $\beta$ Pictoris $b$; the values of $R^{2}$ are associated only with the regression performed using the central values for the mass and radius of $\beta$ Pictoris $b$.

While, as expected, both $\Pi$ and $R K E$ produce extremely accurate bilogarithmic regressions with the mass of the planets, as well as the percentages of the explained variance are practically identical, on the basis of (7) and (8) only the rotational kinetic energy turns out to be accurately proportional to the square mass of the planets.

This result appears hardly due to chance, quite the same way as the general trend of planetary spin with mass [3]; rather, it can be suggestive of a general physical mechanism such as the one described in Sect. 3.

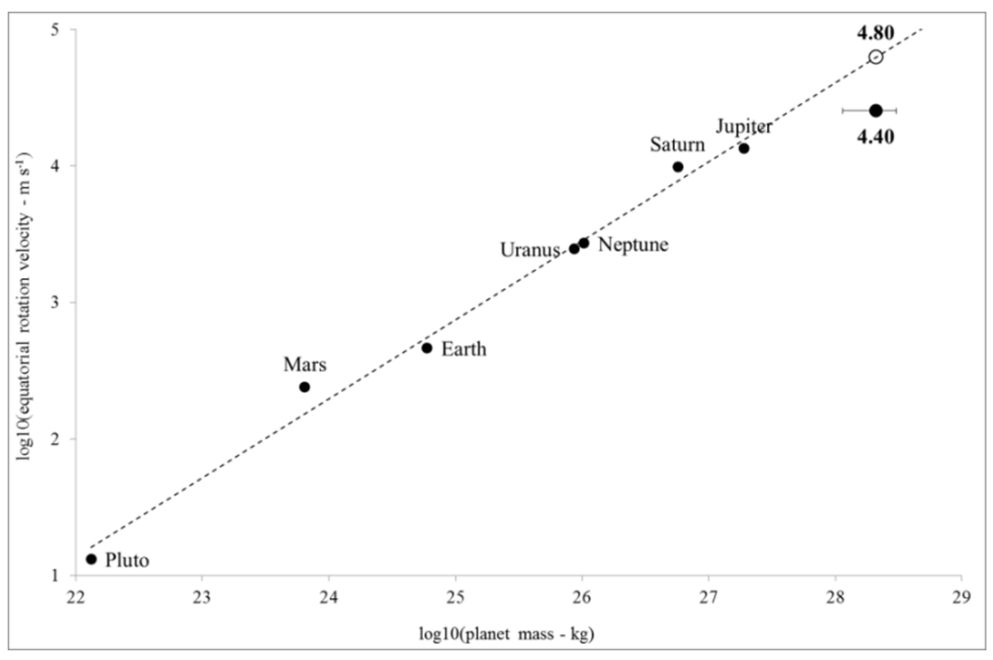

(a)

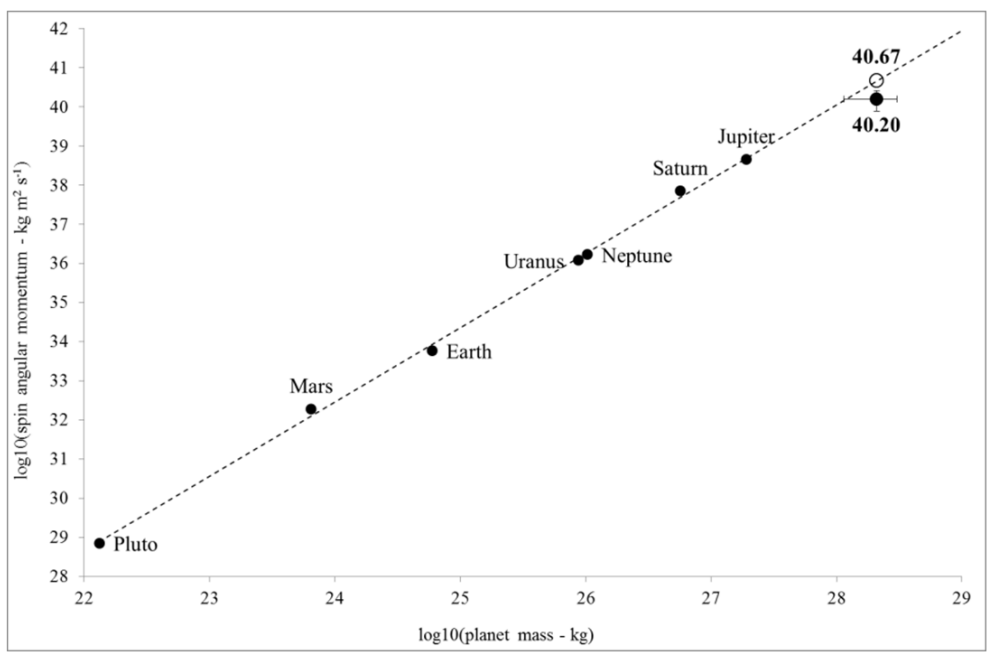

(b) 


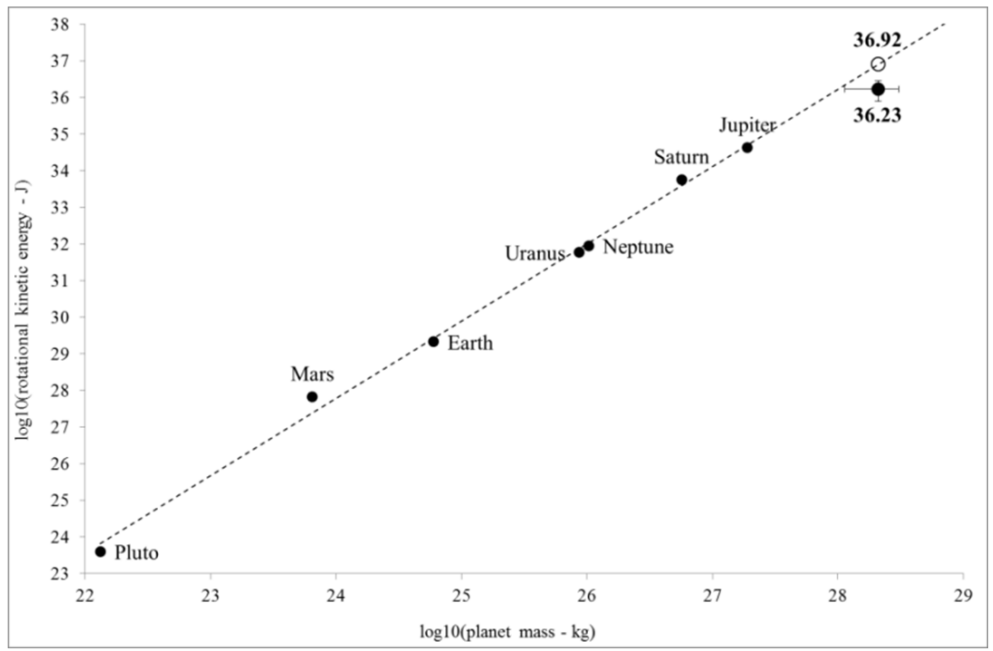

(c)

Fig. 1. Predictive ability of the bilogarithmic regressions of different expressions for the planetary spin with the mass for Solar System planets with regards to $\beta$ Pictoris $b$ in turn represented by open (predicted) and filled (observed) circles on the upper right side of each diagram: (a) ERV, (b) $\Pi$, (c) $R K E$

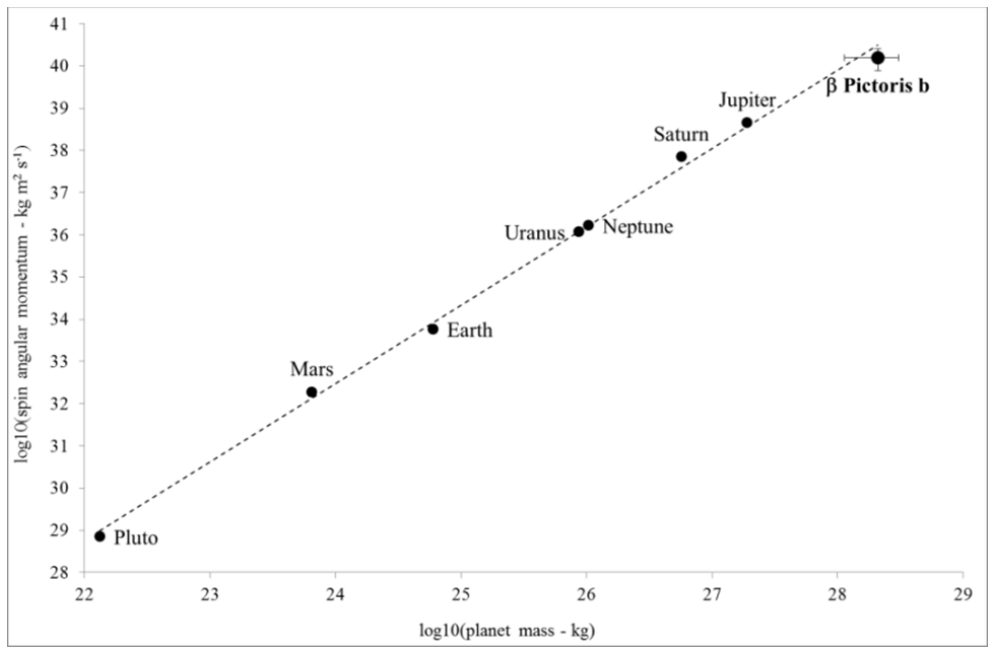

(a)

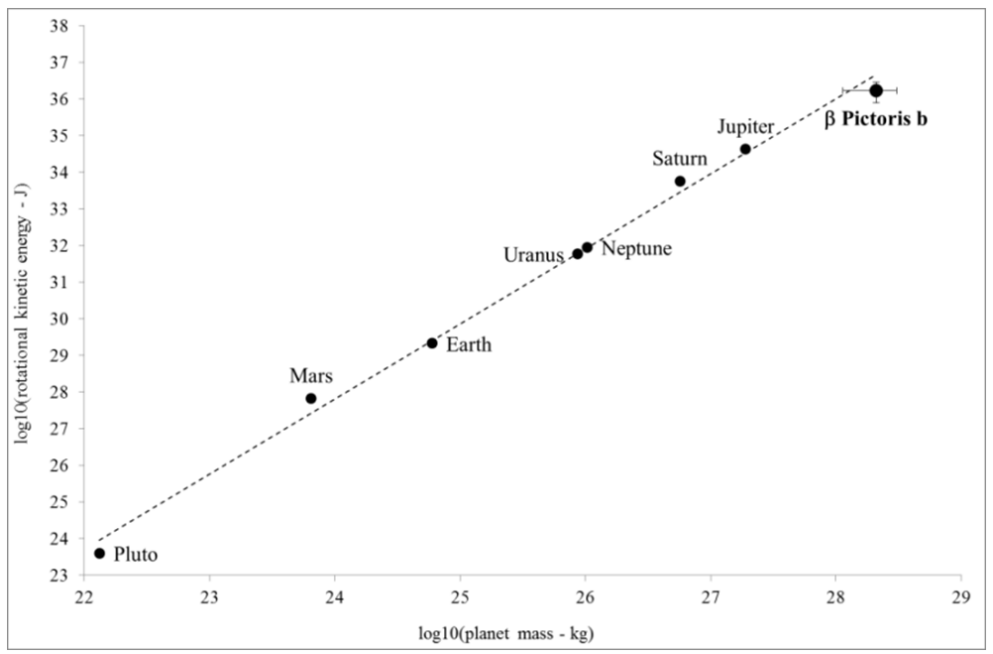

(b)

Fig. 2. Bilogarithmic regressions of different expressions for the planetary spin with the mass for Solar System planets and $\beta$ Pictoris b: (a) $\Pi$, (b) RKE 
Actually, the observed close proportionality of the rotational kinetic energy of a planet to its square mass (8), although it can possibly be challenged in the future by further measurements of spin for other exoplanets, turns out to be quite in agreement with the prediction performed on the basis of the theory of byuons (6), which is an original finding of this article.

\section{Conclusions}

The arguments dealt with in this article provide at least some clues towards the explanation of the observed accurate proportionality of the rotational kinetic energy to the square of the mass of planets in the Solar System and beyond.

Nevertheless, much theoretical work remains to be done, as well as more comprehensive verification is needed; one of the key points appear to be the scale analysis of possible contributions to the rotational energy coming from the as yet partially unsolved long series development of the complex wave functions describing the byuons' interactions (e.g. equation (19) and equation (20) in [13]).

Therefore, while no definitive conclusion can be drawn here, a direction for further investigation is identified and, in turn, the observed accurate proportionality of the rotational kinetic energy with the square of the mass of planets revealed in (8) could be useful in order to step forward the analysis and consequences of the theory of byuons, while understanding that other more classical physical mechanisms able to explain the same result, such as described in [1], cannot be ruled out at this stage.

Furthermore, only gathering further data including at least the mass, the equatorial radius and the spin (e.g. the equatorial tangential velocity), of other exoplanets that are unaffected by large potentials of force fields different from the gravitational one as well as that are far enough from their stars in order to avoid relevant tidal interactions, will allow an increasingly significant verification of the predictive capabilities of the byuon theory with regards to the planetary rotational kinetic energy.

In any case, the fact that the achieved results agree with the predictions of the byuon theory further supports not only the investigation of other natural systems and phenomena, but also the design and development of new technologies such as described in [24-27], to be carried out on the basis of the same physical theory.

\section{Acknowledgments}

F. Zabini and M. Pagliaro are gratefully acknowledged for suggestions and continuous support in communication style.

\section{References}

[1] Hughes DW. Planetary spin. Planet Space Sci 2003;51:51723.
[2] Snellen IAG, Brandl BR, de Kok RJ, Brogi M, Birkby J, Schwarz H. Fast spin of the young extrasolar planet $\beta$ Pictoris b. Nature 2014;509:63-5.

[3] Barman T. Astronomy: A new spin on exoplanets. Nature 2014;509:41-2.

[4] Yoder CF. Astrometric and Geodetic Properties of Earth and the Solar System. In: Ahrens TJ, editor. Glob. Earth Phys., vol. i, Washington, D. C.: American Geophysical Union; 1995, p. 1-31.

[5] Rubincam DP. Pluto and Charon: A case of precession-orbit resonance? J Geophys Res 2000;105:26745-55.

[6] Chauvin G, Lagrange A, Beust H, Bonnefoy M, Boccaletti A, Apai D, et al. Orbital characterization of the $\beta$ Pictoris $b$ giant planet. Astron Astrophys 2012;542:1-9.

[7] Baurov YuA, Timofeev IB, Chernikov VA, Chalkin SF, Konradov AA. Experimental investigations of the distribution of pulsed-plasma-generator radiation at its various spatial orientation and global anisotropy of space. Phys Lett A 2003;311:512-23.

[8] Baurov YuA. Space magnetic anisotropy and a new interaction in nature. Phys Lett A 1993;181:283-8.

[9] Baurov YuA, Klimenko EYu, Novikov SI. Experimental observation of space magnetic anisotropy. Phys Lett A 1992;162:32-4.

[10] Baurov YuA. The anisotropic phenomenon in the $\beta$ decay of radioactive elements and in other processes in nature. Bull Russ Acad Sci Phys 2012;76:1076-80.

[11] Baurov YuA. Research of global anisotropy of physical space based on investigation of changes in $\beta$ and $\alpha$-decay rate of radioactive elements, motion of pulsars and anisotropy of cosmic rays. Am J Mod Phys 2013;2:177-84.

[12] Malov IF, Baurov YuA. The distribution of space velocities of radio pulsars. Astron Reports 2007;51:830-5.

[13] Baurov YuA, Malov IF. On the Nature of Dark Matter and Dark Energy. J Mod Phys 2010;01:17-32.

[14] Baurov YuA. The Anisotropy of Cosmic Rays and the Global Anisotropy of Physical Space. J Mod Phys 2012;03:1744-8.

[15] Baurov YuA, Spitalnaya AA, Abramayan AA, Solodovnikov VA. Seismic activity of the earth, the cosmological vectorial potential and method of a short-term earthquakes forecasting. Nat Sci 2011;03:109-19.

[16] Baurov YuA, Malov IF, Meneguzzo F. Tornadoes and the global anisotropy of the physical space. Am J Mod Phys 2014;3:93-112.

[17] Baurov YuA, Konradov AA, Kushniruk VF, Kuznetsov EA, Sobolev YuG, Ryabov YuV., et al. Experimental investigations of changes in beta-decay rate of $60 \mathrm{Co}$ and 137Cs. Mod Phys Lett A 2001;16:2089-101.

[18] Baurov YuA. On the structure of physical vacuum and a new interaction in Nature (Theory, Experiment and Applications). NY: Nova Science; 2000.

[19] Baurov YuA. Global Anisotropy of Physical Space, Experimental and Theoretical Basis. NY: Nova Science; 2004. 
[20] Baurov YuA, Malov IF. Variations of Decay Rates of Radioactive Elements and their Connections with Global Anisotropy of Physical Space. Int J Pure Appl Phys 2010;6:469-482. Also at: http://arxiv.org/abs/1001.5383.

[21] Baurov YuA, Sobolev YG, Ryabov Y V., Kushniruk VF. Experimental investigations of changes in the rate of beta decay of radioactive elements. Phys At Nucl 2007;70:182535 .

[22] Sturrock PA, Buncher JB, Fischbach E, Javorsek II D, Jenkins JH, Mattes JJ. Concerning the Phases of the Annual Variations of Nuclear Decay Rates. Astrophys J 2011;737:65.

[23] Baurov YuA. Nature of Inertia forces. Int Lett Chem Phys Astron 2014;10:14-24.
[24] Baurov YuA, Meneguzzo F, Baurov AYu, Baurov AYuJ. Plasma Vacuum Bubbles and a New Force of Nature, The Experiments. Int J Pure Appl Sci Technol 2012;11:34-44.

[25] Baurov YuA, Baurov AYu, Baurov AYuJ, Meneguzzo F, Bugaev AA. New Interaction in Nature and its Use in the Form of Traction. Int J Pure Appl Sci Technol 2012;13:40-9.

[26] Baurov YuA, Albanese L, Meneguzzo F, Menshikov VA. Universal propulsion harnessing the global anisotropy of the physical space. Am J Mod Phys 2013;2:383-91.

[27] Baurov YuA, Albanese L, Meneguzzo F, Menshikov VA. Protecting the Planet from the Asteroid Hazard. Int J Pure Appl Phys 2013;9:151-68. 